\title{
Enhancement of Mobility Model for Cluster Hierarchical Routing Protocol in Wireless Sensor Networks
}

\author{
N. M. Nawar, S. E. \\ Soliman,N.M.Ayad \\ EAEA, Cairo, Egypt.
}

\author{
H. S. El-Sayed \\ Electrical Engenering dept. Faculty of \\ Electronic Engineering, Menoufia \\ University,Shebin El-Kom, Egypt
}

\author{
M.H. Kelash \\ Computer Science and Engineering \\ dept. Faculty of Electronic \\ Engineering, Menoufia University, \\ Menouf, Egypt
}

\begin{abstract}
The recent researches in wireless sensor in nuclear applications are studying the usage of wireless sensor network and how utilize and maximize its benefits in our installations in Egyptian Atomic Energy of Authority (EAEA).

The communication protocols named LEACH is a clustering based protocol in which the nodes organize themselves into local clusters, with one node acting as the cluster head [1-5]. This paper present simulation results that illustrate enhancement of LEACH protocol and the importance of choosing a mobility model in the simulation of Wireless sensor Network [6-8]. Specifically. Also, illustrate how the performance resultsof WSN drastically change as a result of enhancement of LEACH simulated by obtaining the distance and energy information from advertise message .Network simulator ( NS2) will be used to prove that after enhancement performs better than LEACH protocol and enhance average Energy, Delay and Network life time for Random Way mobility Model.
\end{abstract}

\section{Keywords}

Wireless sensor networks; cluster; Routing Protocol; Simulation; Performance Comparison; Mobility Model

\section{INTRODUCTION}

New mobile and wireless protocols are hot areas for researchers to support novel applications and to enhance the overall performance of wireless sensor network amongst mobile nodes. Energy-saving and network life time are an essential for nodes operation, effective energy and resource management has become an important part of the design issues to be considered, so , research on energy consumption has attracted more and more attention [9]. Leach is one of the most popular hierarchical (cluster based) routing protocol in order to minimize the energy consumption; in this paper we propose an improvement clustering on the Leach Protocol that further enhance the Power consumption. Assuming transmission power control is available, sensor nodes in the original LEACH choose their clusters based on the received signal strength of the cluster heads' announcements. A sensor node determines the distance to all communicated cluster heads and joins the cluster with a nearest cluster head. The cluster head aggregates data from all the cluster members and sends the aggregate data to the base station (BS). Such procedure requires the least power to communicate with the cluster head. However, the energy consumption at the cluster head is not considered. Since sensor nodes are initially deployed by random way, the node distribution is irregular which leads to that a cluster has many members and other has a few members. The cluster head with many members deplete its energy rapidly. Accordingly, this distance-based approach is not completely optimal to prolong the network life time; so, some intermediate nodes are lost.

This paper focus in enhancement LEACH protocols using in our consideration energy and distance to choose best cluster head [10].

Simulations of wireless sensor network employ several components critical to the accuracy of the simulations, Random Way Point being the choice of mobility model in this paper.

The rest of paper is organized as follow: section 2 present, the related work, section 3 provide application, Section 4present LEACH protocols, Section 5 describes briefly the concept of Structure Analysis of LEACH after enhancement Algorithm, Section 6 introduce briefly Random Way Point, in Section 7 proves the after enhancement algorithm through experiments and analyzes the experiment results, and finally, the conclusion is drawn in Section 8.

\section{RELATED WORKS}

Currently, there are many network protocols for WSNs with their own advantages and disadvantages. The Directed Diffusion [11] is a protocol eliminates redundancy of data and minimizes the number of transmissions; thus saving energy and prolonging network lifetime and advantage is a gradient to describe the possibility of network intermediate nodes continuing their search for matched data along the direction. The disadvantage is that there are no multiple routes that carry data to the sink node, and the routing robustness is not good enough. The LEACH \& LEACH_C protocols: LEACH is a cluster-based protocol with distributed cluster formation the algorithm randomly elects cluster heads and rotates the role to distribute the consumption of energy. Its proposer later improved it, the author in [12] proposing the LEACH_C The main enhancement was through the clustering nodes no longer compete for cluster heads, but nodes first send their own data to the sink node, and then the sink node determines the position of cluster heads according to their location, energy and cycle. The advantage of doing so lies in that we get a reasonable distribution of clusters through a reasonable arrangement of cluster heads, reducing the energy consumption due to the non-ideal random location or numbers of clusters in the original LEACH algorithm. For now, the LEACH_C and LEACH protocols may be considered generally equivalent hierarchal cluster routing protocols. [13] proposed an algorithm that handle the fault tolerant and reliability requirements for data communication in sensor networks, so in this approach each node has a maximum transmission range to avoid link failure due to the loss power of sensor nodes (small battery) and it uses Total Time to Live (TTL) to adjust the level of data reliability, so that the more TTL the more possible routes used by the data the more robust it 
would be in the case of link or node failures. In [14] a low energy adaptive clustering hierarchy (LEACH) has been proposed which divided the nodes into clusters, in each cluster a dedicated node with extra authority called cluster head $(\mathrm{CH})$ is responsible for creating and manipulating a TDMA (time division multiple access) schedule and sending aggregated data from nodes to the base station where these data is needed using CDMA (code division multiple access). Remaining nodes are cluster members until next election .

\section{APPLICATION}

The applications for WSNs describe in tracking, monitoring and controlling. WSNs are mainly utilized for object tracking, nuclear reactor control, fire detection, and traffic monitoring. Intrusion system is a common application in Radioisotope production plant (RPF) in Inshas site at EAEA, in which the sensor networks is deployed over a region where some incident is to be monitored. For example, a large quantity of sensor nodes could be deployed inside the project to detect enemy intrusions When the sensors detect the event being monitored (heat, pressure, sound, light, electro-magnetic field, vibration, etc.), the event needs to be reported to one of the base stations (control room), which can than take some appropriate action. Wireless sensor networks are used extensively within the water/wastewater industries. Facilities not wired for power or data transmission can be monitored using industrial wireless I/O devices and sensor nodes powered by solar panels or battery packs. Wireless sensor networks can use a range of sensors to detect the presence of vehicles for vehicles detection. Wireless sensor networks are also used to control the temperature and pressure levels inside ventilation system. When the temperature and pressure drops below specific levels, the maintenance responsible can be notified via e-mail or a cell phone text message, open vents, turn on fans, or control a wide variety of system responses. Because some wireless sensor networks are easy to install, they are also easy to move when the needs of the application change or on case of accident.

\section{LEACH PROTOCOLS}

In $\mathrm{LEACH}$, the nodes organize themselves into local clusters, with one node acting as the cluster head [15]. All non-clusterhead nodes must transfer their data to the cluster-head, while the cluster head node must receive data from all the cluster members, perform signal processing functions on the data (e.g., data aggregation), and transmit data to the remote base station (BS). Therefore, being a cluster-head node is much more energy-intensive than being a non-cluster-head node [16].

\subsection{Structure Analysis of Routing protocol after enhancement Algorithm}

The proposed routing mechanism, after enhancement, similar to the original LEACH divides the nodes into clusters, each containing a cluster head whose task is considerably more energy-intensive than the rest of the nodes. Accordingly, nodes rotate tasks at different rounds between a cluster head and normal sensors throughout the lifetime of the network. The length of each round is chosen such that each node is expected to be able to perform a cluster head task once during its lifetime. At the beginning of each round, each sensor node, not chosen as a cluster head before, makes an independent decision through a randomized algorithm about whether or not to assume a cluster head task. Nodes that choose to be cluster heads announce their status to the rest of the network. Based on the received signal strength of these announcements, sensors in the original LEACH join the cluster that requires the least power to communicate with the cluster head (assuming transmission power control is available). The cluster head aggregates data from all the cluster members and sends the aggregate data to the base station. However, there is no interaction between nodes when the cluster heads are chosen. Therefore, the cluster heads may be chosen such that there is no uniformity throughout the network. In addition, certain sensors are forced to join clusters located at large distances from them. The after enhancement solves those problems which a new algorithm is designed to choose the best cluster heads. In the original LEACH, nodes choose the best cluster head according to the least distance for all cluster heads. The cluster head's distance can be obtained from the advertise message that the cluster head broadcasts for all nodes in the network. Afterwards, each node makes a list for communicated cluster heads and chooses its cluster head with least distance. After enhancement algorithm considers the distance and the remaining energy for the cluster head.

So the proposed method designs its decision variable (d) as follows:

$$
\begin{gathered}
d_{i j}=\sqrt{\left(\left(\alpha S_{i j}\right)^{2}+\left(E_{i}-E_{m}\right)^{2}\right.} \\
\alpha=\frac{\Delta E_{a v g}}{\Delta S_{a v g}}
\end{gathered}
$$

Where $E_{i}$ Is the initial energy, $E_{m}$ is the remaining energy of cluster head $\mathrm{j}, S_{i j}$ is the estimated distance between the nodes $\mathrm{i}$ and the cluster head $\mathrm{j}$, Sensitivity parameter $(\alpha)$ is add to sense the small change of the estimated distance and the consumed energy, $\left(E_{i}-E_{m}\right.$ ), is defined as the ratio between the average of the first order differences of the consumed energy and the average of the first order differences of the estimated distance. And $d_{i j}$ is the node i decision value for cluster head $\mathrm{j}$.

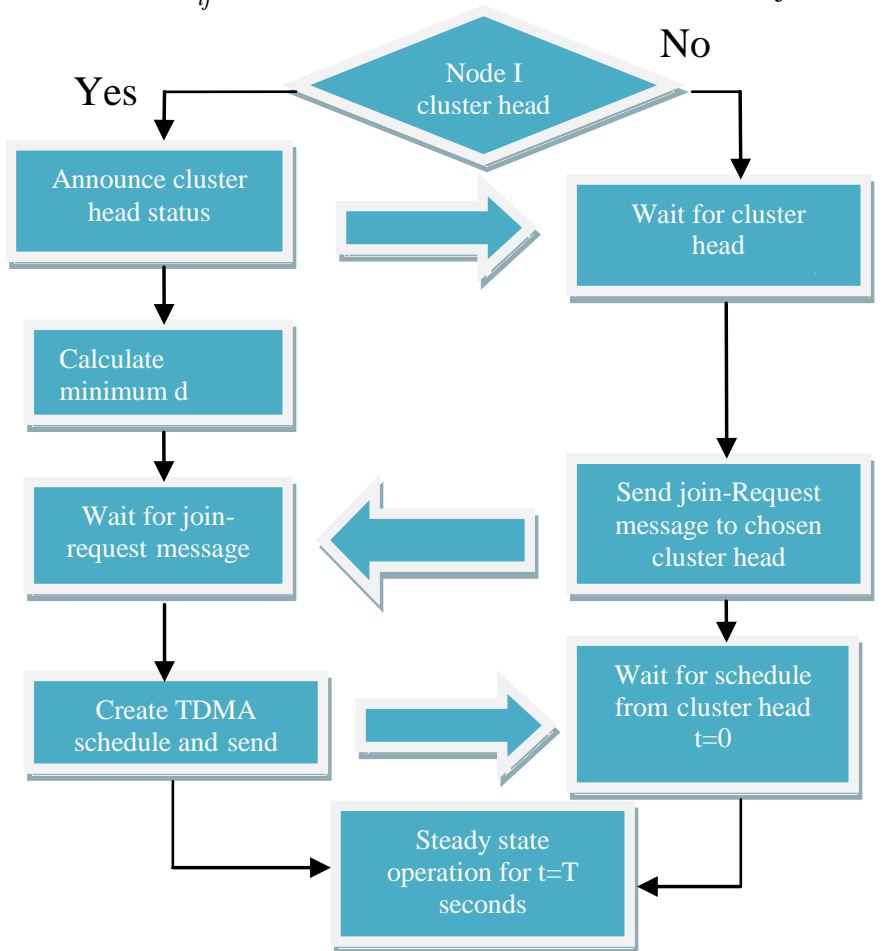

Fig.1 Flow-graph of the distributed cluster formation algorithm After Enhancement 


\section{RANDOM WAY POINT}

The random waypoint mobility model introduces specific pause times for mobility node in wireless sensor networks between movements changes in direction and speed ( velocity )[17] in this model, Once this pause time expires, the mobility node chooses a random destination as well as a speed that is uniformly distributed between zero \& maximum and chose anew destination quickly. The main drawback, Memory-less movement state (a common problem for all random waypoint variations).

\section{SIMULATION EXPERIMENTS AND RESULTS}

Conventionally, the wired sensor network in Egyptian Atomic Energy of Authority (EAEA) in RPF project is used to sense temperature and pressure in ventilation system. In this paper, we establish enhancement in LEACH routing protocol to provide wireless sensor network will be used in hot area .So, the specification of network grid is $600 \times 600$ meters. The nodes are randomly distributed and the movement is generated by Bonnmotion [18] .The base station is placed at the origin of the network topology, at location $\mathrm{x}=0, \mathrm{y}=0$. All sensor nodes start with a fixed amount of Initial Energy $10 \mathrm{j}$. Numbers of nodes are $(10,25,50,75,100,150,200,250,300$, and 350). Data size is 1000 byte. Transmission range is $250 \mathrm{~m}$. Traffic source is Temperature app. Packet size is 500 byte. Transmit power is 0.395 w. Receiving power is $0.660 \mathrm{w}$. Number of clusters is $20 \%$ from no of nodes. Reception energy consumption is $24 \mathrm{mw}$. Routing protocol is LEACH - after enhancement and Simulation time is 600 second. The performance of protocol after enhancement is complied with LEACH-MAE (LEACH-Mobil Average Energy based) [19]. The performance is evaluated mainly, according to the following Metrics.

- Packet delivery ratio.

- Average Delay.

- Average Energy Ratio.

- Network Life Time.

- Throughput.

- Overhead.

\subsection{Random waypoint based performance comparisons}

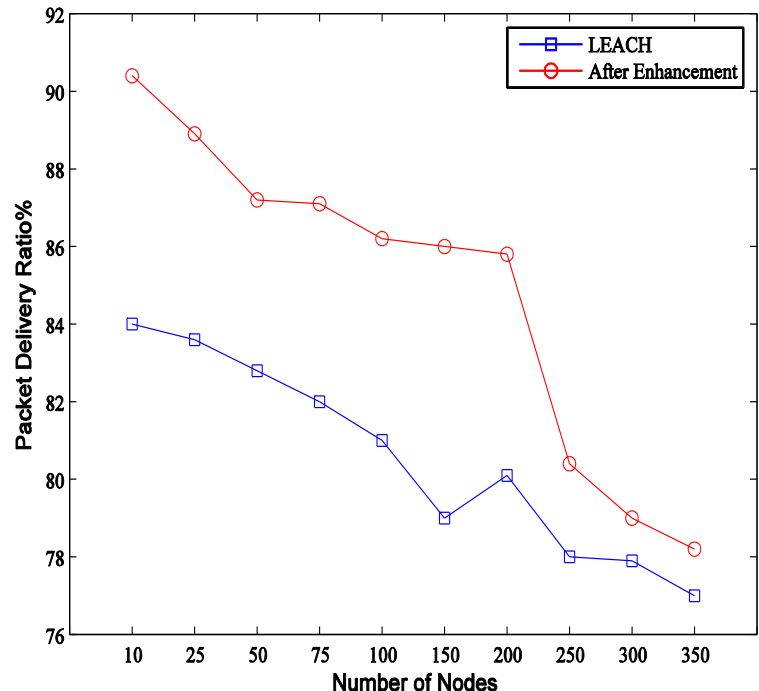

Fig.2 Packet delivery ratio at Random Way Point mobility mode
Figure 2 shows the results of different simulation experiments to measure packet delivery ratio at different numbers of sensor nodes for the LEACH and after enhancement. The minimum packet delivery ratio occurred at 350 sensor nodes at which the ratio is $78 \%$ for LEACH and $80.4 \%$ for after enhancement, while the maximum packet delivery ratio occurred at 10 sensor nodes at which the ratio is $84 \%$ for LEACH and $90.4 \%$ for after enhancement Therefore, on the average, we can realize that AFTER ENHANCEMENT can improve the packet delivery ratio compared with the LEACH.

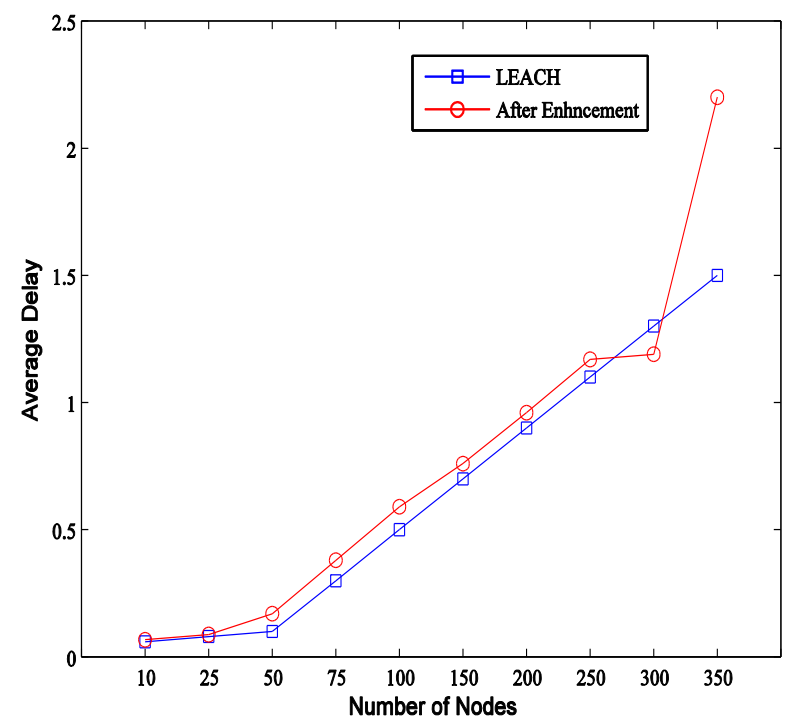

Fig.3 Average Delay at Random Way Point mobility model

Figure 3 shows the results of different simulation experiments to measure Average Delay at different numbers of sensor nodes for the LEACH and after enhancement. The minimum Average Delay occurred at 10 sensor nodes at which the ratio is $0.06 \mathrm{~s}$ for LEACH and $0.068 \mathrm{~s}$ for after enhancement, while the maximum Average Delay occurred at 350 sensor nodes at which the ratio is $1.1 \mathrm{~s}$ for $\mathrm{LEACH}$ and $1.17 \mathrm{~s}$ for after enhancement Therefore, on the average, we can realize that after enhancement can improve the Average Delay compared with the LEACH.

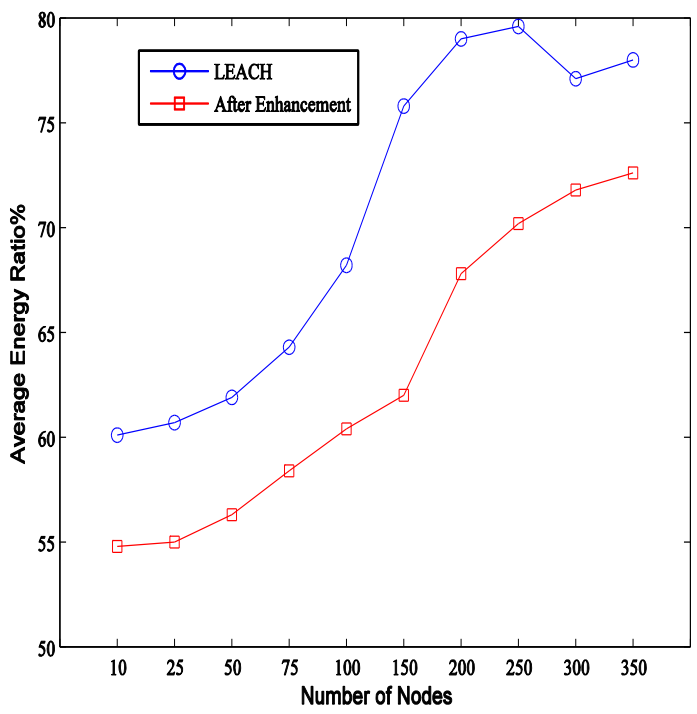

Fig.4 Average energy ratio at random way point mobility model 
Figure 4 shows the results of different simulation experiments to measure Average Energy Ratio at different numbers of sensor nodes for the LEACH and after enhancement. The minimum Average Energy Ratio occurred at 10 sensor nodes at which the ratio is $63.8 \%$ for LEACH and $61 \%$ for after enhancement, while the maximum Average Energy Ratio occurred at 350 sensor nodes at which the ratio is $87 \%$ for LEACH and $82 \% \mathrm{~s}$ for after enhancement Therefore, on the average, we can realize that after enhancement can improve the Average Energy Ratio compared with the LEACH.

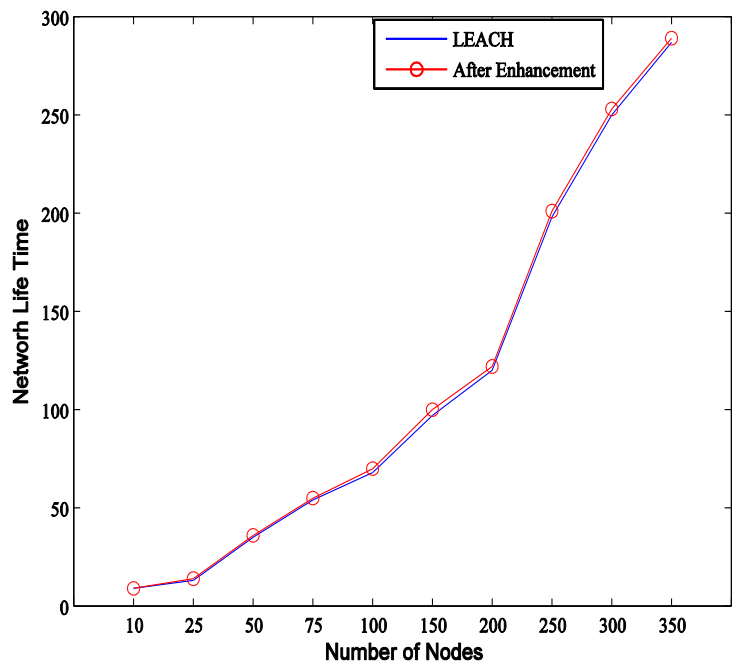

Fig.5 Network lifetime at Random Way Point mobility model

Figure 5 shows the results of different simulation experiments to measure Network Life Time at different numbers of sensor nodes for the LEACH and after enhancement. The minimum Network Life Time occurred at 10 sensor nodes at which the ratio is 9 for LEACH and $9 \mathrm{~s}$ for after enhancement, while the maximum Network Life Time occurred at 350 sensor nodes at which the ratio is 189 for LEACH and 201 for after enhancement Therefore, on the average, we can realize that after enhancement can prolong the network lifetime compared with the LEACH.

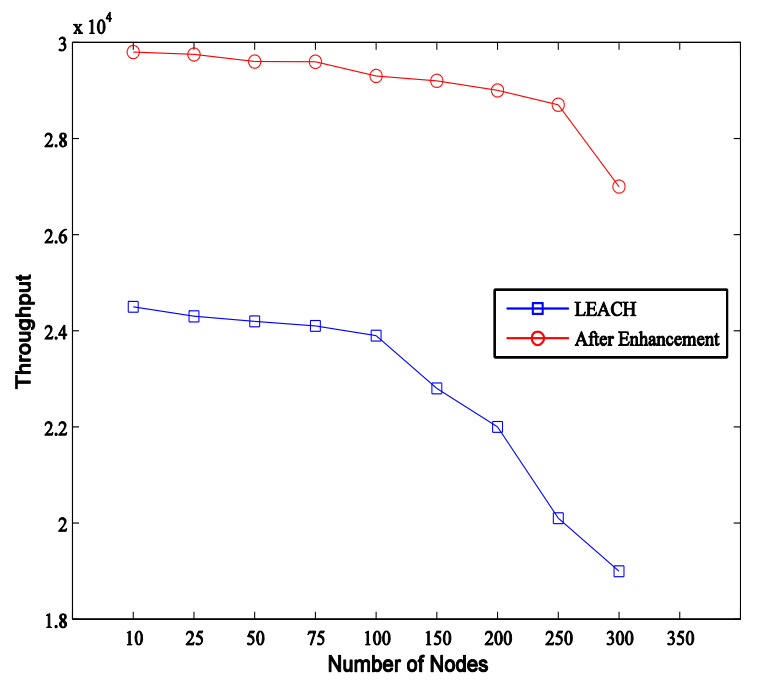

Fig.6 Throughput at Random Way Point mobility model

Figure6 shows the results of different simulation experiments to measure throughput at different numbers of sensor nodes for the LEACH and after enhancement. The minimum throughput occurred at 350 sensor nodes at which the ratio is 20100 for LEACH and $28700 \mathrm{~s}$ for after enhancement, while the maximum throughput occurred at 10 sensor nodes at which the ratio is 24500 for LEACH and 29800 for after enhancement.

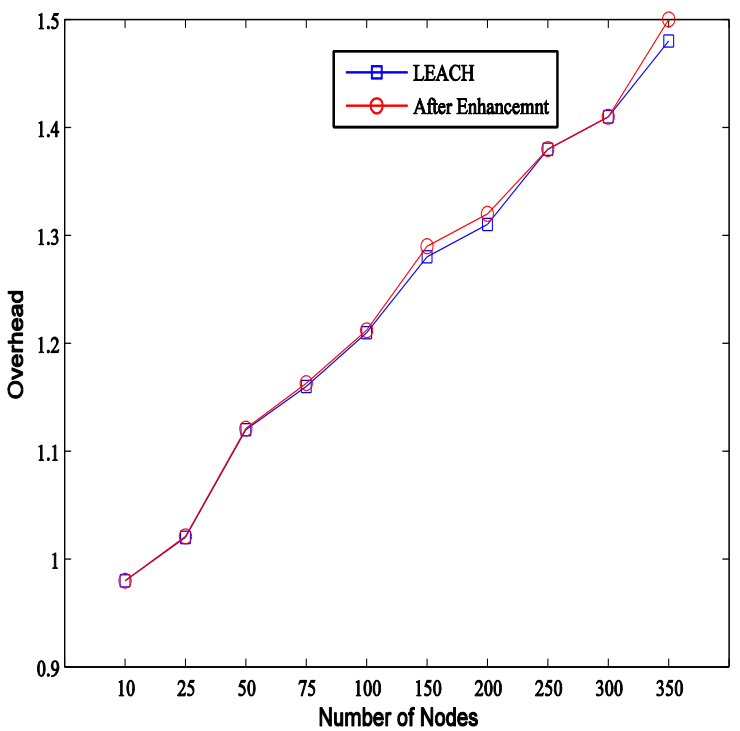

Fig.7 Overhead at Random Way Point mobility model

Figure7 shows the results of different simulation experiments to measure overhead at different numbers of sensor nodes for the LEACH and after enhancement. The minimum overhead occurred at 10 sensor nodes at which the ratio is 98 for LEACH and 98 for after enhancement, while the maximum overhead occurred at 350 sensor nodes at which the ratio is 1.38 for LEACH and1.38 for after enhancement.

\section{CONCLUSION}

In this paper, introduction of LEACH protocol for wireless sensor networks which use clusters to manage the communications between different sensor nodes and the base station, then we mentioned the most shortcomings in this protocol and proposed a new version of LEACH which is suppose to solve such shortcomings, the proposed protocol is called after enhancement. As the original LEACH fails to achieve an internal balance because the energy consumption clearly differs from a cluster head to another in the same round.

This paper introduce the enhancement of LEACH protocols and how to choose best cluster head and apply this on Random Way Point mobility model .comparison simulation prove that LEACH protocol after enhancement improve network life time, packet delivery ratio, energy consumption, average delay and throughput.

We also conclude that the simulation results verified and validated with standard specifications. Also, a nuclear application that is based on sensor was presented, implemented and simulated using NS2 simulator

\section{REFERENCES}

[1] O. Younis, M. Krunz and A. Ramasubramanian, "Node Clustering in Wireless Sensor Networks: Recent Developments and Deployment Challenges", IEEE Network, May-June-2006, pp. 20-25. 
[2] A. Abbasi and M. Younis, "A survey on clustering algorithms for wireless sensor networks", Elsevier Sci, J. Computer Communications, Vo1.30,2007, pp. 2826-2841.

[3] C. Liu, C. Lee, L.ChunWang, "Distributed clustering algorithms for data gathering in Wireless mobile sensor networks", Elsevier Sci. J. Parallel Distrib. Comput., Vol.67, 2007, pp.1187-1200

[4] N.Kim, J.Heo, H.Kim and W. Kwon, "Reconfiguration of cluster heads for load balancing in wireless sensor networks" Elsevier Sci. J. Compo Communications, Vol. 31,2008, pp. 153-159

[5] P. Tillaport, S. Thammarojsakul, T.Thumthawatworn and P. Santiprabhob, "An Approach to Hybrid Clustering and Routing in Wireless Sensor Networks", In Proc. IEEE Aerospace, 2005, pp. 1-8

[6] C. Tracy, B. Jeff, D.Vanessa, "A Survey of Mobility Models for Ad hoc Network Research. Special Issue on Mobile Ad-hoc Networking: Research, Trends and Applications," Journal of Wireless Communications and Mobile Computing, 2002, pp. 483-502.

[7] Fan Bai and Ahmed Helmy. A Survey of Mobility Models in Wireless Adhoc Networks. Proceedings of IEEE information Communication Conference (INFOCOM 2003), April 2003.

[8] Kumar, M V, and Jacob. Mobility Metric based LEACHMobile Protocol. ADCOM 2008, IEEE 2008.

[9] Jiang, J.A.; Chen, C.P.; Chuang, C.L.; Lin, T.S.; Tseng, C.L.; Yang, E.C.; Wang, Y.C. CoCMA: Energy-Efficient Coverage Control in Cluster-Based Wireless Sensor Networks Using a Mimetic Algorithm. Sensors 2009, no. 9, pp.4918-4940

[10] M. Sajjad, W. Daniel, M. Stefan, Position-based Routing Protocol for Low Power Wireless Sensor networks. Journal of Universal Computer Science, vol. 16, no. 9, pp.12151233,2010

[11] Akkaya, K.; Younis, M. A Survey on Routing Protocols for Wireless Sensor Networks. Ad. Hoc. Networks 2005, no.3, pp.325-456.

[12] Liu, B.; Wang, L.; Jin, Y. Advances in Differential Evolution. CHIN. J. Control Decision 2007,vol.22,pp.721729.

[13] Jen-Yeu Chen, Yen-Shiang Shue, Hakeem Ogunleye, Saurabh Bagchi, A comparative study on data fault tolerant requirements for data propagation in sensor networks, 2003.

[14] Stephanie Lindsey, Cauligi S. Raghavendra, PowerEfficient Gathering in Sensor Information Systems (PEGASIS).
[15] K. Atta Ur Rehman, M. Sajjad, H. Khizar, K. Samee Ullah,"Clustering-based power-controlled routing for mobile wireless sensor networks. International Journal of Communication Systems (IJCS)," vol. 4, no. 25, 2012, pp. 529-542, 2011.

[16] Jennifer Yick, Biswanath Mukherjee, Dipak Ghosal . Wireless sensor network survey. Elsevier Science 2008.

[17] J. Broch, D. A. Maltz, D. B. Johnson, Y.-C. Hu, and J. Jetcheva, A performance comparison of multi-hop wireless ad hocnetwork routing protocols, in Proceedings of the Fourth Annual ACM/IEEE International Conference on Mobile Computing and Networking(Mobicom98), ACM, October 1998

[18] BonnMotion, "A Mobility Scenario generation and analysis tools", University of Bonn, pp. 4-14, Nov. 2011.

[19] Abbirah Ahmed and Sameer Qazi , “ Cluster Head Selection Algorithm for Mobile Wireless Sensor Networks", National University of Sciences and Technology (NUST), pp. 120-125, International Conference on Open Source Systems and Technologies (ICOSST) 2013.

\section{AUTHOR'S PROFILE}

NadiaM.Nawar.Cairo,14 Dec.1984,BS.communication and electronics engineering,Soubra faculty of engineering, Benha university,EL-Qalubia, 2006. Eng. Dept., HOT LAB Center, EAEA, Inshas, Abo-zaable, Cairo, Egypt,.

Hamdy M. Kelash received the Eng. Degree from the Institute of Electronic Engineering, Egypt in 1971, MSc degree from Faculty of Engineering Technology, Helwan University, Egypt, in 1979 and the PHD degree from Institute National Polytechnique (INP), France in 1984. He has been lecturer in 1984 at the Electronic Industry department, Faculty of Electronic Engineering, also a lecturer in 1987 at the Computer Sciences and Engineering department, and an Assistant Professor in 1993 and the Head of Computer Sciences and Engineering department, Faculty of Electronic Engineering, Menoufia University from 2001 to 2007. His main research interests include optical computing, artificial intelligence, network security, image processing, digital systems and parallel computing.

Hala S. El-sayed received the B.Sc., M.Sc., and Ph.D. degrees in electrical engineering from Menoufia University, Shebin Elkom, Egypt, in 2000, 2004, and 2010, respectively. She is currently with the Department of Electrical Engineering, Faculty of Engineering, Menoufia University, where she was a Demonstrator from 2002 to 2004 and has been a Lecturer since 2010. Her research interests cover Network security, Wireless sensor network, Secure building automation systems, and Biometrics.

Prof. Soliman, Egyptian Atomic energy Authority Former chairman of HLWMC. MSc , PhD - Danmarks Tekniske Universitet - DTU BSc - faculty of engineering - Elazhar Uni 